\title{
Celebrating diversity to promote and create inclusive curricula
}

Authors: Naomi Gostelow, Amali Lokugamage, Faye Gishen

Institution: University College London Medical School

Corresponding author: Dr Naomi Gostelow iBSc MBBS PGCert FHEA

Address: Rm 138 Medical School Building

74 Huntley Street

WC1E 6BT

Email: n.gostelow@ucl.ac.uk

Tel: 07762419437

\section{Co-Authors: Miss Amali Lokugamage MBChB BSc MSc MD FRCOG SFHEA \\ Dr Faye Gishen BSc MBBS FRCP SFHEA}

Contributions: Naomi Gostelow assisted in producing the educational innovation as a symposium including presenting at and documentation of the event. Amali Lokugamage applied for funding for the mapping exercise and symposium and produced the symposium. Faye Gishen as the academic lead took an advisory role during production of the teaching innovation and presented at the symposium. All authors contributed to the write up and gave their consent for the article to be submitted.

Conflicts of interest: none to declare

Funding: the student mapping project and symposium were funded by the University College London Liberating the Curriculum fund.

Ethical considerations: No major ethical issues were encountered. The work constituted an audit of current practice and symposium event and so did not fulfil requirements for formal ethical approval by the UCL research ethics committee.

Acknowledgements: We would like to thank all academic and administrative staff members who made this educational initiative possible and the UCL Arena Centre and Liberating the Curriculum working group for providing inspiration and support.

Word count: 500 
Celebrating diversity to promote and create inclusive curricula

\section{Celebrating diversity to promote and create inclusive curricula}

\section{What problem was addressed?}

Promoting diversity within medical curricula is a priority from both medical governing bodies and institutions serving increasingly diverse populations (1). However, evaluating how diversity is taught and learned can be difficult and often confused with staff and student equality resulting in a tick-box exercise (1). Diversity should be taught in a way which engages students helping them to collaborate with patients and approach them compassionately and holistically (1). This may be best performed in an integrated way, which is aligned with a pan-college initiative called "Liberating the Curriculum" (LTC).

\section{What was tried?}

We aimed to celebrate current work promoting diversity in the medical school and identify areas for improvement. First and second year students were recruited to map the curriculum identifying sessions which incorporated the following themes: discrimination, social class, ethnicity, gender, sexuality, disability, stigmatised groups, patient experience, human rights and challenging power hierarchies. Students analysed educational materials for each year group available on the institution's virtual learning environment and evaluated where these themes were addressed. The results were presented at a student-staff collaborative LTC symposium. Staff already promoting the diversity agenda were also invited to present their work. The event culminated in a student panel and open discussion addressing what was done well and how these priorities can be applied to other parts of the curriculum. The student panel used this discussion as a springboard for future innovations and will produce a manifesto to promote sustainability and a medical school-wide commitment to tackle these issues throughout the curriculum.

\section{What lessons were learned?}

The student mapping exercise showed a high representation of these diversity themes particularly within the first three years of the medical curriculum with patient experience the most highly represented. The 
Celebrating diversity to promote and create inclusive curricula

last two clinical years contained much less of this type of material. The symposium drew great interest from both internal stakeholders and external institutions many commenting that the content had inspired new insights. This forum allowed for teachers already promoting diversity to be given a voice. As an intervention it allows both educators and students to challenge prior assumptions and assimilate new knowledge to transform their teaching materials in line with societal change. The student panel are currently producing their manifesto to further promote diversity in the curriculum. Suggestions include providing images of clinical signs in the context of different backgrounds, for example dermatological conditions in different skin tones, culturally appropriate actors and scenarios for practical assessments, decolonisation of the curriculum and acknowledging racial and gender discrimination in development of medical knowledge. These priorities need to be amplified in the latter part of the curriculum prior to students exiting for postgraduate practice. The student panel has created foundations for a formal student group to perpetuate the LTC agenda. This group is currently performing a qualitative analysis on the symposium's feedback for a greater understanding of its impact. The symposium reflected that it is only by recognising and discussing these issues, challenging preconceptions and acknowledging unconscious biases that we can create truly inclusive curricula.

\section{Reference}

1. Turner MA, Kelly M, Leftwick P, Dogra N. Tomorrow's Doctors and diversity issues in medical education. Med Teach. 2014 May; 36:9, 743-745 